

\author{
MIT Open Access Articles
}

SPHERES flight operations testing and execution

The MIT Faculty has made this article openly available. Please share how this access benefits you. Your story matters.

Citation: Mohan, Swati, Alvar Saenz-Otero, Simon Nolet, David W. Miller, and Steven Sell. "SPHERES Flight Operations Testing and Execution." Acta Astronautica 65, no. 7-8 10ctober 2009): 1121-1132.

As Published: http://dx.doi.org/10.1016/j.actaastro.2009.03.039

Publisher: Elsevier

Persistent URL: http://hdl.handle.net/1721.1/99453

Version: Author's final manuscript: final author's manuscript post peer review, without publisher's formatting or copy editing

Terms of use: Creative Commons Attribution-Noncommercial-NoDerivatives 


\author{
IAC-07-A2.6.03 \\ SPHERES Flight Operations Testing and Execution \\ Swati Mohan \\ Graduate Student, Massachusetts Institute of Technology, Cambridge, MA USA \\ smohan@mit.edu \\ Dr. Alvar Saenz-Otero \\ Research Associate, Massachusetts Institute of Technology, Cambridge, MA USA \\ alvarso@mit.edu \\ Dr. Simon Nolet \\ Post-Doctoral Associate, Massachusetts Institute of Technology, Cambridge, MA USA \\ snolet@mit.edu \\ Dr. David W. Miller \\ Professor, Massachusetts Institute of Technology, Cambridge, MA USA \\ millerd@mit.edu \\ and \\ Steven Sell \\ SPHERES Project Manager, Payload Systems Inc., Cambridge, MA USA \\ sell@payload.com
}

\begin{abstract}
$\underline{\text { Abstract }}$
SPHERES (Synchronized Position Hold Engage Reorient Experimental Satellites) is a formation flight testing facility consisting of three satellites operating inside the International Space Station (ISS). The goal is to use the long term microgravity environment of the ISS to mature formation flight and docking algorithms. The operations processes of SPHERES have also matured over the course of the first seven test sessions. This paper describes the evolution of the SPHERES program operations processes from conception to implementation to refinement through flight experience. Modifications to the operations processes were based on experience and feedback from Marshall Space Flight Center Payload Operations Center, USAF Space Test Program office at Johnson Space Center, and the crew of Expedition 13 (first to operate SPHERES on station). Important lessons learned were on aspects such as test session frequency, determination of session success, and contingency operations. The paper describes the tests sessions; then it details the lessons learned, the change in processes, and the impact on the outcome of later test sessions. SPHERES had very successful initial test sessions which allowed for modification and tailoring of the operations processes to streamline the code delivery and to tailor responses based on flight experiences.
\end{abstract}

\section{Introduction to SPHERES}

The SPHERES project, Synchronized Position Hold Engage Reorient Experimental Satellites project, is a formation flight testbed operating inside the International Space Station (ISS) developed by the Massachusetts Institute of Technology (MIT). The objective is to provide a fault tolerant environment to perform cutting edge research for algorithm development in fields such as estimation, docking, formation flight, reconfiguration, fault detection and path planning.

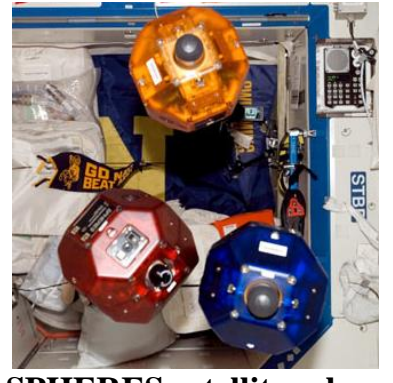

Figure 1: SPHERES satellites aboard the ISS

Aboard the ISS, SPHERES consists of three identical satellites, a laptop computer ground station, and a radio frequency (RF) transmitter to communicate between the laptop and the satellites. The SPHERES position and attitude determination system (PADS) uses a combination of infrared (IR) flash, to 
synchronize their clocks, and ultrasonic time-offlight measurements to triangulate their position in a pseudo-GPS system. The SPHERES satellites operate within an approximately $2 \mathrm{~m}$ by $2 \mathrm{~m}$ by $2 \mathrm{~m}$ volume [1].

SPHERES satellites run on $\mathrm{CO}_{2}$ propellant at 25 psi, for an effective $I_{\text {sp }}$ of 32 seconds. The satellites are powered by two battery packs, consisting of eight AA batteries each. Each satellite also has an expansion port, which allows for external payloads to be attached at a future date [2].

The ISS configuration is replicated at the MIT Space Systems Laboratory in Cambridge, MA. Three ground satellites are maintained for testing of algorithms prior to launch.

\section{SPHERES History}

SPHERES was originally developed as part of a undergraduate design class at MIT in 1999. The objective was to give undergraduates the experience of the entire lifecycle of the development of an aerospace product, teaching the engineering process as "conceive, design, implement, and operate” (CDIO). Thirteen undergraduate students worked to develop functional requirements beginning in February 1999, with fully integrated prototype hardware ready a year later. The satellites were tested aboard the KC-135 Reduced Gravity Aircraft in the spring of 2000 in short duration microgravity testing.

The design of the CDIO class was updated and the flight satellites were developed, as a joint effort with Payload Systems Inc. Three satellites were scheduled to be delivered to NASA in June 2003, with an expected launch date of July 2003. After the Columbia accident in February 2003, the team projected a delay of a few months.

The first SPHERES hardware, consisting of one beacon and beacon tester, was launched on Progress 12P. This was used in Expedition 8 by astronaut Michael Foale to test the ultrasound and infrared environment of the US lab. This activity was performed twice, once in November 2003 and again in March of 2004.

All satellite hardware was delivered in August 2003 with the expectation of launch in November on Progress 13P and January 2004 on 14P. The manifest for $13 \mathrm{P}$ was two satellites, with the third on 14P. The SPHERES hardware was de-manifested from 13P, and then from 14P; the hardware was returned to MIT in November 2003. NASA's objective was to maximize science with minimum mass. The SPHERES team had multiple iterations of manifest opportunities over the next three years in order to send up partial SPHERES hardware to accomplish some minimized science objectives. However, none of these opportunities came to fruition. The first SPHERES satellite (Red) was launched aboard flight Progress 21P on April $24^{\text {th }}$, 2006. Also launched was a communications transmitter, along with several batteries and tanks. By 2006, the SPHERES team had shrunk from eight to six members, with only four original members remaining.

The first test sessions occurred less than a month after launch, on May $18^{\text {th }}$ and May $20^{\text {th }} 2006$, operated by Expedition 13 astronaut Jeff Williams. The second satellite (Blue) launched aboard STS-121 (ULF1.1) on July 4 2006. The third and final satellite (Orange) launched on December $10^{\text {th }}$, 2006 on board STS-116 (12A.1). SPHERES hardware was took four launches to arrive on the ISS, and over three years longer than the originally assumed few months.

Table 1 highlights the test session dates and the astronaut operating SPHERES. Table 2 gives a description of all astronauts trained on SPHERES, when they trained, and when they operated SPHERES on the ISS, if applicable. Table 2 shows the time delay between the training and operations for the crew.

Table 1: SPHERES Test Session Dates

\begin{tabular}{|l|c|c|c|}
\hline Session & Date & Exp & Crew \\
\hline TS001 & $5 / 18 / 2006$ & 13 & J. Williams \\
\hline TS002 & $5 / 20 / 2006$ & 13 & J. Williams \\
\hline TS003 & $8 / 12 / 2006$ & 13 & J. Williams \\
\hline TS004 & $8 / 19 / 2006$ & 13 & J. Williams \\
\hline TS005 & $11 / 11 / 2006$ & 14 & M. Lopez-Alegria \\
\hline TS006 & $3 / 17 / 2007$ & 14 & $\begin{array}{c}\text { S. Williams / M. } \\
\text { Lopez-Alegria }\end{array}$ \\
\hline TS007 & $3 / 24 / 2007$ & 14 & S. Williams \\
\hline TS008 & $4 / 27 / 2007$ & 14 & S. Williams \\
\hline
\end{tabular}

Table 2: Crew Training Dates vs Flight Dates

\begin{tabular}{|l|l|l|l|}
\hline \multicolumn{1}{|c|}{ Name } & Expedition & Trained & On ISS \\
\hline Mike Barrat & N/A & July 2002 & Dry Run \\
\hline Mike Foale & Exp 8 & Oct 2003 & \\
\hline
\end{tabular}




\begin{tabular}{|l|l|l|l|}
\hline Mike Fincke & $\begin{array}{l}\text { Exp 9 } \\
\text { Exp 13 B/U } \\
\text { Exp 16 B/U }\end{array}$ & $\begin{array}{l}\text { Jan 2004 } \\
\text { Jan 2006 } \\
\text { May 2007 }\end{array}$ & \\
\hline Dan Tani & $\begin{array}{l}\text { Exp B/U } \\
\text { Exp 16 }\end{array}$ & $\begin{array}{l}\text { Aug 2004 } \\
\text { May 2007 }\end{array}$ & \\
\hline Leroy Chiao & Exp 10 & Aug 2004 & \\
\hline Bill McArthur & $\begin{array}{l}\text { Exp 10 B/U } \\
\text { Exp 12 }\end{array}$ & $\begin{array}{l}\text { Aug 2005 } \\
\text { Mar 2005 }\end{array}$ & \\
\hline John Phillips & Exp 11 & Aug 2005 & \\
\hline Clay Anderson & $\begin{array}{l}\text { Exp 11 B/U } \\
\text { Exp 15* }\end{array}$ & $\begin{array}{l}\text { Mar 2005 } \\
\text { May 2006 }\end{array}$ & \\
\hline Jeff Williams & Exp 13 & Mar 2005 & May 2006 \\
\hline Suni Williams & $\begin{array}{l}\text { Exp 13 } \\
\text { Exp 14* }\end{array}$ & $\begin{array}{l}\text { Mar 2005 } \\
\text { May 2006 }\end{array}$ & Mar 2007 \\
\hline Leo Eyhart & ESA B/U & Apr 2006 & \\
\hline Thomas Reiter & Exp 13 ESA & Apr 2006 & \\
\hline $\begin{array}{l}\text { Mike Lopez- } \\
\text { Alegria }\end{array}$ & Exp 14 & May 2006 & Nov 2006 \\
\hline Peggy Whitson & Exp 13 & May 2006 & \\
\hline $\begin{array}{l}\text { Gregory } \\
\text { Chamitoff }\end{array}$ & Exp 15 B/U & Dec 2006 & \\
\hline $\begin{array}{l}\text { Sandra } \\
\text { Mangus }\end{array}$ & Exp 17 & May 2007 & \\
\hline
\end{tabular}

* Indicates refresher training

\section{Test Session Description}

\subsection{Original Concept of Operations}

The concept of operations during SPHERES development, up until the spring of 2006, was fairly abstract. The team had requested to be onsite at Johnson Space Center (JSC) for the first two test sessions, in order to have live coverage. It was assumed that live coverage would only be available for the first two sessions, and perhaps for critically important sessions afterwards, such as the first three satellite operations. All other test sessions may have real-time off-site support from the SPHERES team, but no direct interaction or communications. Originally, it was envisioned that SPHERES would operate with an approximate rate of a test session every two weeks. The first test session was expected to occur with one satellite, the second with two satellites, and three satellite operations by the third test session.

A usability test was conducted in 2002 with astronaut Michael Barrat. This testing confirmed the usability of the Graphical User Interface (GUI), and provided an early assessment of useful information for the crew during test execution, such as title, objective, description, and possibly a short video demonstration. It was understood that the team would be able to upload a memo with instructions for that particular test session. This was thought to be the only communication with the crew prior to the test session. The SPHERES team did not have a formalized plan from delivery to operations, but instead was reactionary and flexible to what the latest news was from NASA. Through the preparation and execution of the first seven test session, a formalized process was developed, executed, and refined.

\subsection{Preparation for Operations}

Preparation for the first test session began roughly a month prior to session date. The two main aspects were algorithm and operations preparation. Algorithm preparation consisted of creating the SPHERES Program File (SPF). The SPF file contains the project file with the algorithms, html files for an overview of each test, and a text file to link the tests from the graphical user interface (GUI) to a test command to the satellite. The first test session SPF delivery to DOD Space Test Program (STP) was on April $28^{\text {th }}$.

Operations preparation included the development of contingency plans and processes for the SPHERES team, practice session on the ground with retired astronaut Jeff Hoffman, and a crew conference with astronaut Jeff Williams prior to the first test session.

For contingency operations, the team employed two methods of preparation, a listing of possible anomalies with corresponding visual/audio symptoms and a flow diagram of possible test outcomes. The list of possible anomalies was used to perform a "greencard" session. During this session, the ops team receives a symptom ("greencard") given in terms of an audio of visual cue. The team then must plan the responsive action within a given time limit. This was set up to mimic cues and time frames that would be typical during the test session.

For the mock test session, Hoffman was given the SPHERES procedures and asked to perform the test session. He walked through the set-up and execution of the test session as it would occur in space. This mock test session was useful in catching errors in procedures, as well as to highlight areas that might cause issues during the actual operations.

The third method of operations preparation was to refresh the astronaut in the operations of SPHERES. Astronaut Jeff Williams was trained on SPHERES one year prior, in March 2005. Due to this long gap between training and 
operations, it was critical to refresh the crew's training. The refresh training consisted of prepared slides and a telecon with the crew prior to the test session. The telecon was conducted on Tuesday May $16^{\text {th }}$. This direct conversation between the astronaut and the SPHERES program manager provided an opportunity for last-minute reminders from the SPHERES team, and a chance for the crew to ask questions or clarifications.

\subsection{TS001}

Test Session 1 (TS001) was performed on May $18^{\text {th }}, 2006$ by astronaut Jeff Williams. The technical objective was to verify that the hardware was working properly. The science objectives were to demonstrate the basics of formation flight and autonomous docking. TS001 included general tests of open and closed loop control, beacon tracking, and beacon docking.

TS001 was successful in demonstrating the proper operation of the hardware, and open loop control. An error in the gyroscope scaling factors prevented the science objective of the session being achieved. Due to fast acquisition of the data, rapid analysis and code development enabled a fix to be included in the next test session. This file was uploaded to the ISS the next day in preparation for the following test session on May $20^{\text {th }} 2006$.

\subsection{TSO02}

Test session 2 (TS002) occurred on May 20 ${ }^{\text {th }}$, 2006, also was operated by astronaut Jeff Williams. Guest scientists from NASA Ames Research Center (ARC) performed mass identification and fault detection tests, while the MIT SPHERES team performed tests on formation flight and docking.

The preparation for the test was done jointly with TS001, with original delivery of the SPF also on May $11^{\text {th }}$. The updated session code was uploaded on May $19^{\text {th }}$, containing a test to fix the error observed in TS001. The test demonstrated the errors observed in TS001 were successfully corrected.

\section{$\underline{3.5 \text { TS003 }}$}

TS003 was performed on August $12^{\text {th }}, 2006$ by astronaut Jeff Williams. The technical objective was to perform a hardware checkout of the second satellite. The science objectives were to perform docking, path planning, mass identification, and initial two satellite operations.

Preparation was done jointly with TS004, and was limited to algorithm preparation. Since the astronaut was familiar with SPHERES, no conference call was held prior to the test session. The SPHERES team was given about two weeks notice prior to the session. There had been three potential opportunities earlier in the summer, so the prepared files were updated periodically. TS003 contained three SPF files, two from MIT and one from the guest scientist at NASA ARC, which were all delivered one week prior to the test session.

\section{$\underline{3.6 \text { TSO04 }}$}

TS004 was performed on August 19 ${ }^{\text {th }}$, 2006 and was the final test session performed by astronaut Jeff Williams. The science objective was to test the global navigation system and the global metrology set-up. This included a procedure for the crew to set-up the wall mounted beacons, which had not been performed to date. Though the astronaut was experienced operating SPHERES, the set-up of the global metrology system turned out to be confusing, primarily because of the graphics in the GUI that helps the crew identify the location in which they have placed the beacons. This set-up required significant communication between the crew and the SPHERES ops team to confirm questions and clarify procedures.

As this was the last session for Expedition 13, a crew debrief with astronaut Jeff Williams occurred on November $7^{\text {th }}, 2006$.

\section{$\underline{3.7 \text { TSO05 }}$}

TS005 occurred on November $11^{\text {th }}, 2006$, conducted during Expedition 14 by astronaut Michael Lopez-Alegria. The science objectives for TS005 were mass identification, docking, reconfiguration, and path planning. Since this was the first test session for Lopez-Alegria, the SPHERES team had a crew conference with him on October 25th, 2006.

\section{$\underline{3.8 \text { TSO06 }}$}

TS006 occurred on March $17^{\text {th }}$, 2007. This session was jointly performed by astronauts Michael Lopez-Alegria and Sunita Williams during Expedition 14. The crew conference for astronaut Sunita Williams occurred immediately prior to the test session. The science objectives for this test session were identical to TS005 
because there was only one week notice to the SPHERES team of the session opportunity. The preparation for this test session was minimal because tests prepared for TS005 but not yet executed were run. As such, there was no additional algorithm preparation. An updated test plan was generated and sent to DOD STP prior to the session.

\section{$\underline{3.9 T S 007}$}

TS007 occurred on March 24 $4^{\text {th }}, 2007$ and was performed by astronaut Sunita Williams, though astronaut Lopez-Alegria was still present onboard the ISS. TS007 was the first session with all three satellites. Thus, the technical objective was to confirm proper functionality of the third satellite, as well as simultaneous operation of all three satellites. The science objectives were docking, reconfiguration, lost-inspace recovery maneuvers, and three satellite formation flight.

A debrief for Expedition 14 crew Michael Lopez-Alegria and Sunita Williams was held on April $18^{\text {th }}, 2007$.

\subsection{Summary}

The progress made in operation procedures and process over the course of the seven test sessions is partially captured in Table 3 . Table 3 shows that though the overall number of tests planned for remained fairly constant, the number of tests coded decreased significantly. Later session plan to execute all tests prepared. Also, there has been an increase in the number of tests executed per session. The overhead time (set-up and cleanup) was longest for TS004 and TS005. TS004 was the first session with global metrology, and TS005 was a new astronaut and global metrology set-up. It is also noted that the change of crew does not have a significant impact on the results of the test or the number of tests executed.

Table 3: Test Session Breakdown

\begin{tabular}{|c|c|c|c|c|c|c|c|}
\hline & \multicolumn{7}{|c|}{ Test Sessions } \\
\hline & 1 & 2 & 3 & 4 & 5 & 6 & 7 \\
\hline $\begin{array}{l}\text { Tests } \\
\text { Coded }\end{array}$ & 84 & 45 & 32 & 23 & 26 & $\begin{array}{l}\mathrm{N} \\
\mathrm{A}\end{array}$ & 16 \\
\hline $\begin{array}{l}\text { Tests in } \\
\text { Nominal } \\
\text { Test Plan }\end{array}$ & 21 & 32 & 32 & 23 & 26 & 28 & 19 \\
\hline $\begin{array}{l}\text { Tests } \\
\text { above } \\
\text { "group } \\
\text { success" }\end{array}$ & $\begin{array}{l}\mathrm{N} \\
\mathrm{A}\end{array}$ & $\begin{array}{l}\mathrm{N} \\
\mathrm{A}\end{array}$ & 19 & 15 & 17 & 22 & 17 \\
\hline Tests & 15 & 31 & 24 & 23 & 26 & 27 & 33 \\
\hline
\end{tabular}

\begin{tabular}{|l|c|c|c|c|c|c|c|}
\hline executed & & & & & & & \\
\hline $\begin{array}{l}\text { Session } \\
\text { Duration } \\
\text { (hrs) }\end{array}$ & 3 & 3.2 & 3.5 & 5 & 5.5 & 4 & 4.2 \\
\hline $\begin{array}{l}\text { Total } \\
\text { Test } \\
\text { Time } \\
\text { (hrs) }\end{array}$ & 1.2 & 1.7 & 2 & 2.7 & 2.2 & 2.5 & 3.5 \\
\hline $\begin{array}{l}\text { Avg time } \\
\text { per test } \\
\text { (min) }\end{array}$ & 4.8 & 3.3 & 5 & 5.5 & 5.2 & 5.6 & 6.3 \\
\hline
\end{tabular}

\section{Lessons Learned}

This section describes the lessons learned over the course of the first seven test sessions. The section relates how the operations procedures and processes evolved by learning from the experience of the preparation towards and execution of the first seven test sessions.

\subsection{Test Session Frequency}

The basic objective of SPHERES is to develop and mature algorithms on-board the ISS using an incremental and iterative process. Data from previous test sessions are used to plan and develop algorithms for the next session. In order to close this loop, there must sufficient time between the sessions to allow for data analysis and algorithm development. At the same time, too much time can cause atrophy in the research team, leading to reduced science output. Therefore, the test session frequency has a direct effect on the ability of SPHERES to provide with sufficient and relevant algorithms.

A wide range of periods between test-sessions have been experienced by the SPHERES team. Table 4 summarized these period. Lessons have been learned from all the different time periods so far.

Table 4: Time between Test Sessions

\begin{tabular}{|l|l|l|}
\hline \multicolumn{1}{|c|}{ Period } & Between & \\
\hline 2 days & TS001 & TS002 \\
\hline 1 week & TS003 & TS004 \\
& TS006 & TS007 \\
\hline 3 months & TS002 & TS003 \\
& TS004 & TS005 \\
\hline 4 months & TS005 & TS006 \\
\hline
\end{tabular}

The two day time period between TS001 and TS002 proved to be highly beneficial, and is a recommendation for future programs which 
intend to perform long-duration tests aboard the ISS. The first session, dedicated to hardware checkout, provided the necessary data to the team to determine encountered errors of the facility itself, so that future algorithms could count on a strong foundation. The availability of a test session in the near future enabled the SPHERES team to provide successful scientific achievements in its first round of operations.

The three to four month period between test sessions has been the optimal length of time between sessions from the experience so far. This amount of time enabled the team to fully analyze the data from previous sessions and present the results in formal team reports. Using this data, the SPHERES team was able to iterate on the design of the algorithms, coming up with substantial science advancements for the new sessions. The four month period between TS005 and TS006 was the longest the team would have liked to go without a test session; the four month period was close to causing the team to require raising the importance of other non-ISS tasks, negatively affecting further ISS algorithm development.

The one week time between sessions was the least desired situation for the team. The team was able to take advantage of this situation due to special circumstances. NASA gave only one week warning for new code delivery before TS003 and TS006. Therefore, the team was able to quickly create test plans based on unused tests from TS002 and TS005 in the development of the test plans for TS003 and TS006 respectively. This gave the team two weeks to incorporate fully new tests for TS004 and TS007. However, the one-week repetitions were clearly too short to create any iterations in between. Further, the short time put substantial strain on both the research and operations group.

As a result, the SPHERES team reached a compromise with NASA to schedule sessions approximately three to four months in between. The SPHERES team provides NASA with new test plans and program files within the three month period, and is allowed to update them up to one week in advance of a session.

The most important lesson learned is that the optimal scientific iteration period is three to four months. This time is substantially different than the original idea that iterations would occur every two to four weeks.

\subsection{Contingency planning and operations}

In order to prepare for different environmental conditions, several contingency experiments were planned for the first test session, each with different sets of gains, docking parameters, and controllers. Thus, the TS001 program file contained 84 single satellite tests.

The nominal test plan was to execute 22 tests. In order to determine which test had the appropriate parameters, the astronaut was to be asked to call down at prescribed intervals. The operations team would specify what group to conduct based on the results of preceding tests. Figure 2 shows a flow diagram of the test plan. It shows the large tree structure developed to try to account for algorithm failures at different points during the tests session. After each group, a decision among the different branches was needed. This flow diagram was also communicated in the test plan, in case there should be communication losses at any critical points during the test session.

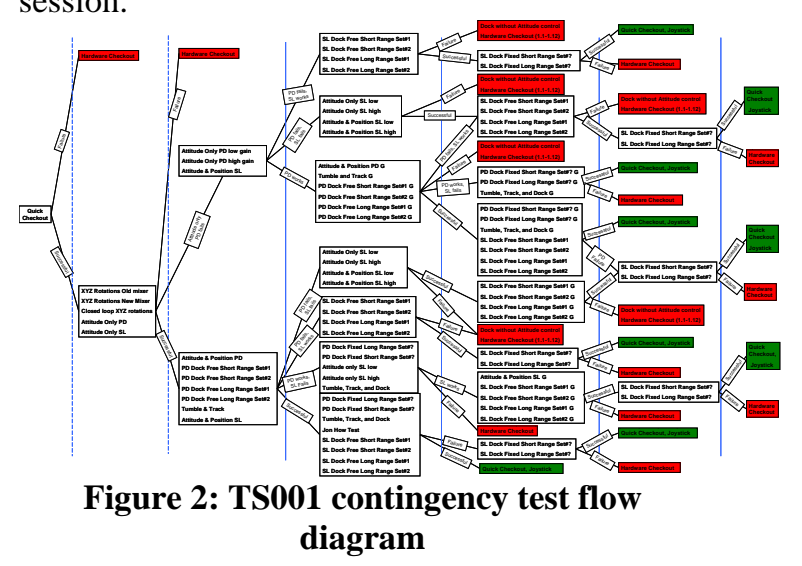

The test plan was 25 pages long; it contained the 40 groups mentioned above. Each group of test had hyperlinks indicating the nominal groups to follow and alternative groups, depending on individual test results. The objective of the hyperlinks was to provide easy navigation through the document, since the exact sequence of groups was not known. The nominal group allowed the crew to proceed in the event of nominal operations or loss of signal, while the alternate groupings provided a method to adapt the tests conducted to best fit the results seen.

The astronaut was to call down at the completion of the penultimate test with the results so far. This allowed the ops team approximately five minutes to determine which group should be run next. Figure 3 is an excerpt of the test plan that 
shows the nominal and alternate groups. The links to later groups, both nominal and contingency, is circled.

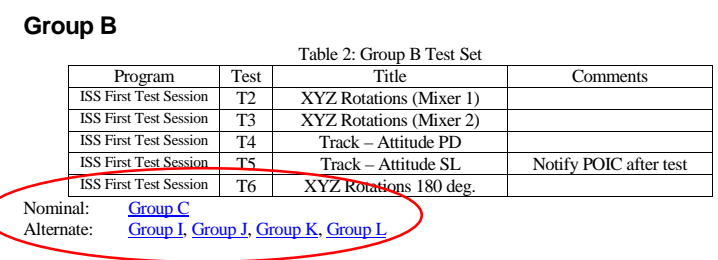

\section{Figure 3: Excerpt from Test Plan of TS001, groups hyperlinks circled}

Despite the complexity of the flow diagram, many options were not captured. During the execution of the test session, results showed that while open-loop rotations worked, closed-loop control did not work. The flow diagram prescribed going straight to a hardware checkout. However, open-loop rotations would not have worked if there had been a hardware failure. Thus, the usefulness of such large flow diagram for nominal operations preparation was suspect. There were too many options to enumerate on a flow diagram. Given real-time audio and video link with the ISS, real-time reactionary analysis was a more effective solution.

The amount of test groups and contingency operations presented to the crew was overwhelming. It was difficult for the crew to obtain the amount of information requested in the test plan in order to make a decision. When there was a decision point, the crew would need to call down for confirmation of a course of action. Due to the complexity of the tests, even with single-satellite operations, and the high availability of interaction between the SPHERES team and the crew, the additional pre-planning was unnecessary.

From the execution of the first test session, it was evident that the symptoms during test execution are such that it is not practical to have the crew decide what path to take. They were capable of analyzing if the positioning was incorrect or if the test did not complete, but they did not determine courses of action based on expected performance. Thus, this experience moved the operations ideology away from one where the astronaut actively helps to analyze tests, to one where they perform a simpler set of sequential tests. Contingency operations became reactionary to questions or comments by the crew, rather than a pre-planned ordering.
The original intent of only having live coverage for the first test session was voided when MIT established its own Payload Operations Center. With this facility at MIT, the SPHERES team has access to NASA video and audio during each test session. Thus, they are more capable of responding quickly for contingency operations. An example of contingency operations in later test sessions was the re-ordering of the test plan in TS005 to account for decreased testing time due to the long set-up time.

\subsection{Test Session groups and group success}

Lessons learned from TS001 indicated that the test plan was too convoluted, due to the large number of tests, groups, and interrelation between the groups. In order to streamline the test plan, the overall number of tests was reduced from 84 in TS001, 45 in TS002, and approximately 25 to 35 in all subsequent sessions. Thus, the test plan was significantly shorter and simpler, consisting of only two to four groups. Also, the interrelation between the groups was removed.

Another question that was raised by NASA officials was "what constitutes a successful test session?" NASA wanted to know at what point it was possible to declare a test session a success. In TS001 and TS002, the crew (and NASA) had only received a series of tests to perform. The number of tests planned was always in excess of the allotted time, so that every available minute was utilized. Also, the number of tests coded was more than the number of tests in the nominal plan. This was a disadvantage since completion of all tests was unlikely; test completion could not be used as a measure of success for the session as a whole.

In order to address this question, the SPHERES team created a group success mark. If the tests above this line were competed, the group was to be considered successfully completed. If all groups met success, then the test session was considered a success. This criterion reflects that success is not determined by the satellites executing a coded test precisely as expected. Since the objective of SPHERES is to test innovative algorithms, every test execution will provide useful data, sometimes more so if the test did not perform as expected. Figure 4 shows a group layout with the success mark.

In TS003, the placement of the mark was intended solely to answer NASA's question. 
Starting with TS004, it became a tool for prioritization. Since different groups generally have different science objectives, it is desired to run some tests from each group during a session. The group success marks became used as tools for when to proceed to the next group if time was running short. Those that were above the group success mark had a higher chance of getting run. Thus, current groups list the tests in priority order, with the science critical to that test session above the group success mark.

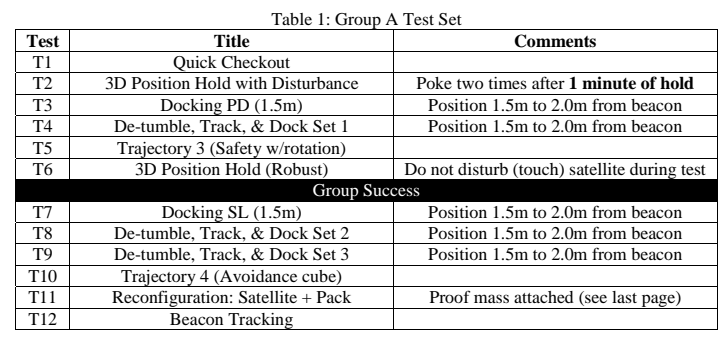

Figure 4: Excerpt from TS003 test plan

\subsection{SPHERES handling on the ISS}

Prior to TS001, there was little to no data about how the environmental conditions on the ISS would affect testing procedures. It was believed that there would be significant drift due to the ventilation system. Therefore, early concepts envisioned the satellites to enter into "positionhold" mode after the astronauts deployed them. However, during TS001, it became evident that there were extremely few disturbances. Thus, the position-hold maneuver was removed, thereby saving propellant and simplifying the software.

As a result of this low disturbance condition, the deployment procedures for one and two satellites are simple. The crew can position the satellites without the need for high accuracy in either position or angle. The satellites remain in place after deployment, and maneuver to their preprogrammed initial conditions with minimal propellant consumption.

However, deployment of three satellites proved to be harder to communicate to the crew. The three-dimensional nature of positioning the three satellites requires $3 \mathrm{D}$ explanations. While multiple methods of communicating set-up have been attempted, such as the use of textual explanations, video, and 2D graphics, none have yet resulted in easy deployment by the crew. Therefore, while new methods to explain 3D deployment to the crew are developed, a simplified approach is taken. For three satellite tests, the test plan utilizes a minimal number of deployment geometries. The crew is clearly told when the same geometry is used in different tests. This requires extended crew time for the first deployment, but minimal time afterwards.

\subsection{Truth sensor requirement}

The major omission in the development of the SPHERES facility is the lack of a truth sensor of higher fidelity than the on-board global metrology [7]. However, the operational design of SPHERES contemplated this by asking NASA to video-tape all of the operations with at least two camera angles. By using perpendicular mounted cameras, the research team could have coarse truth sensing of the three dimensional operations in microgravity.

Apart from the benefits to real-time operations, video data provided the ability to determine correct operations of the global metrology system. To demonstrate the performance of the metrology system, telemetry data was used to super-impose a CAD model of the satellites over a graphic of the ISS with the same perspective as the actual video (Figure 5). Comparison of the two videos demonstrates high accuracy of the global metrology system.

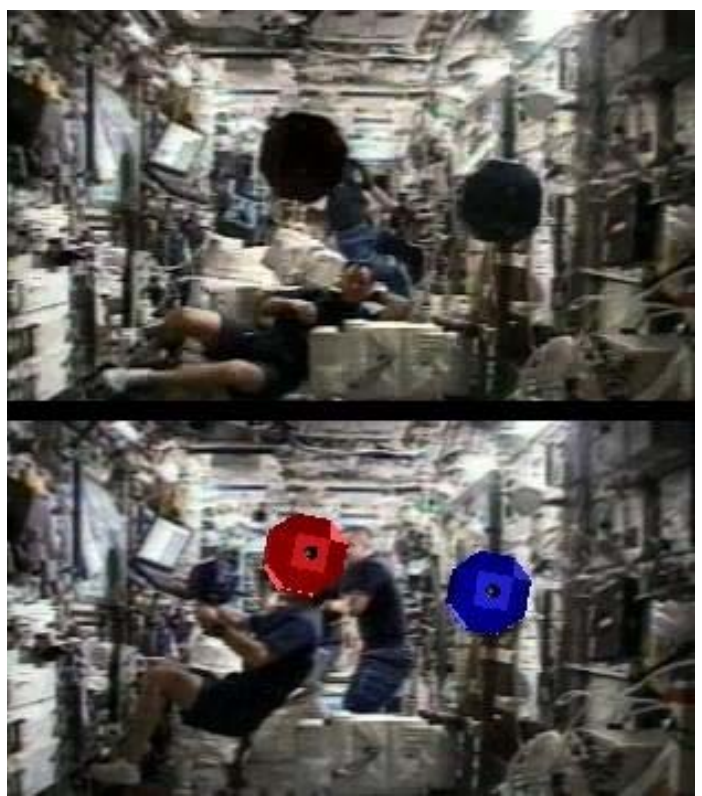

Figure 5: ISS image (top) vs Animation of ISS telemetry in same perspective (bottom)

\subsection{SPHERES set-up procedures}

An important lesson learned was the long learning curve for SPHERES set-up, particularly 
for global metrology. Initial set-up for a new astronaut takes approximately an hour, while an experienced astronaut takes noticeably less time. This was demonstrated in the set-up time between TS001 and TS002, as well as between TS005 and TS006 (Table 3). Global metrology set-up takes an additional hour, even with an experienced astronaut. This was observed in the much longer set-up between TS003 and TS004. This experience led to a request to add an hour to the nominally scheduled operations session to account for the additional time necessary to setup the beacons on all further test sessions.

Another lesson learned about the set-up of the global metrology was that the images provided to identify the beacon locations were confusing. It was unclear that the diagram shown in Figure 6 reflected a 2D projection of that wall. Each blue line corresponds to a seat-track, where the crew was to enter the number of the hole in which the beacon was mounted. Significant explanation was required during TS004 to explain what number to enter and how to interpret the figures. Explanations improved as the SPHERES team gained experience in advantageous locations to place the beacons.

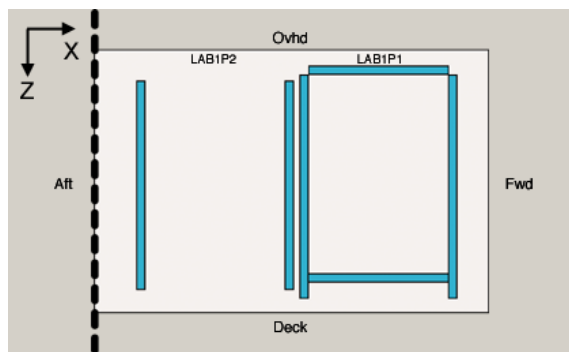

Figure 6: US Lab Port Wall GUI image for entering beacon location

The set-up time was greatly reduced in TS006 because an experienced astronaut, Michael Lopez-Alegria, was present while the new astronaut, Sunita Williams, performed the set-up for the first time. Also, in the execution of the tests, one astronaut would read the test overview and handle the laptop, while the other deployed the satellites. This saved some precious crew time and allowed to perform more tests. Thus, astronaut Sunita Williams was able to come up to speed much quicker than previous astronauts.

\subsection{Communication between crew and team}

The communication interaction with the crew was streamlined significantly over the course of the first few test sessions. Two aspects of importance are noted: communicating to the crew and communication from the crew.

Communication with the crew has greatly been helped by the incorporation of lessons learned. The SPHERES team gained the experience of communicating instructions to the crew. The method of transmitting information from MIT to the crew is from MIT to STP to Paycom at MSFC to the ISS. The experience includes how to phrase instructions succinctly and clearly and when is appropriate to relate the information. Also, experience showed that sometimes it was better to let a test run, in spite of knowing that it may not succeed, for the sake of minimizing complexity in the communication.

Communication from the crew is also very important in enabling real-time contingency operations. When the crew called down a test results number that indicated IR noise, the SPHERES team requested that the lights be dimmed. However, frequent communication from the crew also decreases efficiency. When the crew is without ground communication, they are able to rapidly complete many tests. The longer time per test when in communication could be because of the delay time associated with asking and answering questions. Also, once the crew became more comfortable with the SPHERES testbed, they were able to take more initiative. This limited the communication solely to contingency operations, or for clarifications.

\subsection{Crew Procedures}

The crew procedures for SPHERES were developed in accordance to NASA ISS procedures under the guidance of the Marshall Space Flight Center astronaut office. The procedures offer detailed step-by-step instructions for the setup, operation, and cleanup of the SPHERES hardware and data files during a test session. The unique launch sequence of individual satellites, not contemplated in the original procedures, required special procedures. Further, the lessons learned from the on-going test sessions resulted in changes. As a result, procedures are in a constant state of update.

Lessons learned from crew training and on-orbit test sessions are continuously incorporated to clarify the procedures. NASA has been very accommodating in allowing for fairly frequent revisions. It is clear the NASA MSFC team clearly understands the need for the procedures 
to reflect actual operations. However, experience indicates that configuration control on the version of the procedures is difficult to maintain. This can sometimes lead to difficulties when trying to determine the version the crew is reading. Therefore, future programs should establish ahead of time the ability to have special procedures clearly separate from standard procedures.

The SPHERES procedures have mostly settled to a clear set of standards. Rather than procedural changes, 'special actions' are now uploaded via the test-plan. This allows the SPHERES team to ask the crew for simple tasks without any impact to the overhead. The only reason for procedures to change in the future will be in the addition of new hardware elements or to correct errors.

Another lesson learned is that each astronaut utilizes the procedures in a different manner. Some read them in detail, while others skim. Thus, it is important to find a balance between giving too much detailed information that would unnecessarily prolong operations with too little information which would not be sufficient for session success if the user is simply skimming the procedures. To this end, the team has reworked the information provided to the crew via the GUI and procedures have been streamlined as much as possible. The task continues, as future methods to simplify procedures and minimize overhead for setup continue to be developed.

\subsection{Importance of test result number}

When correcting the gyro scaling factors error in TS002, the test had to be re-run several times until the expected test result value was achieved. The test result number had been coded to inform the team when the scaling factors were set properly. A positive lesson learned on the crew side was of the importance of the test result number. The crew took to calling down the test result number for subsequent tests as well, which greatly helped the ops team to analyze the performance of the test in real-time. This practice was continued by subsequent astronauts as well.

The operations team started to expand the test result numbers to gather more feedback. Most of the tests performed during TS003 were greatly affected by IR noise. However, this information became available only after data analysis. Had this information being available during the test session, the operations team could have ask the crew to take action, like dimming the General Luminescent Assembly (GLA).

Corresponding values were created for errors such as IR noise, beacon location not received, satellite reset, and previous test not completed. For example, the test result for "satellite reset" was observed in TS005 and TS006 and prompted the team to have the crew to change batteries.

\subsection{Difficulty in tank insertion}

The difficulty in the tank insertion was an issue that had been highlighted early on in SPHERES history. In order to determine the impact to operations, the tank insertion was closely watched during the mock test session with Hoffman. As expected, the tank was not inserted fully. Procedures were updated to stress the $3 / 4$ turn past initial resistance, as well as reiterating it at the crew conference. In spite of these efforts, problems occurred when the tank was not fully inserted in TS001. Once the crew became familiar with how to insert a tank, the issue rarely arises again. The tank difficulty is stressed for every new astronaut during crew training, crew conference prior to their first operations of SPHERES on the ISS, as well as in the procedures. The lesson learned was that even if items are stressed multiple times, the lack of the crew to carry out a procedure correctly should not mean mission failure. SPHERES must be resilient to single-point failures in the procedures.

\section{Current Operations Processes}

The current operational procedure begins with the delivery of a program to NASA. Once NASA has a file in hand, it begins to look for a test session opportunity. During this deliberation, the SPHERES team is continuously updating and refining their program.

If SPHERES should be assigned a test session date, a test plan is due to NASA approximately one week prior to the session date. The team also has an opportunity to submit an updated SPF up to a week prior to the session. The file is uploaded onto the ISS laptop during the week of the session. If it is a new astronaut operating SPHERES, the team will have an opportunity to have a telecon with the astronaut prior to the test session for approximately 15 minutes. 
The SPHERES team attends the test session from their operations room at MIT. They are on the phone with the DOD STP representative, as well as having listening access to Payload Operations Center, Space-to-Ground (SG) 1, SG2, and Lead-Increment-Scientist (LIS) loops. The SPHERES team also is able to watch the test session via real-time video downlink from the ISS.

After the test session, the data is downloaded from the ISS laptop to the ground station. It is delivered to MIT within a few days. The DVD of the video from the cameras on the ISS follows a couple of weeks later. Data is analyzed to incorporate into the next session. Results are presented for each test in a report submitted to NASA at the end of each expedition.

\section{Conclusion}

SPHERES was designed to enable the maturation of algorithms aboard the ISS. The design requirements specifically called for the availability of a risk-tolerant environment which enables cutting edge research in a wide range of areas. All of the objectives have been met so far. Table 5 shows the wide range of research covered during the first seven test sessions. These span all the topics listed in the mission objective. Furthermore, as the experiences from multiple test sessions have demonstrated, faults in the design have been overcome. Research has been conducted which pushes the limit of algorithms beyond the levels which would be permissible in standard high-risk space environments. Due to the flexibility of both the ISS/NASA hosts and the design of SPHERES, every test session has provided successful science results, even in the presence of operational difficulties. As a result, SPHERES enables tests which demonstrate the limits of the capabilities of the algorithms. Selected results from tests can be found in the references.

Table 5: Science of ISS Test Sessions

\begin{tabular}{|l|l|}
\hline Test Session & \multicolumn{1}{|c|}{ Science Topic } \\
\hline TS001 & Checkout \\
\hline TS002 & Estimation, docking, system-ID \\
\hline TS003 & $\begin{array}{l}\text { Estimation, docking, formation } \\
\text { flight }\end{array}$ \\
\hline TS004 & $\begin{array}{l}\text { Estimation, docking, formation } \\
\text { TS005 }\end{array}$ \\
fS006 & \begin{tabular}{l} 
flight, fault detection \\
\hline TS007
\end{tabular} \\
& detection, path planning \\
\hline
\end{tabular}

The SPHERES operations have gone through several iterations, reaching a stable design. The lessons learned throughout the seven operating sessions resulted in adjustments to procedures to maximize the efficiency of science conducted with SPHERES. Experience in the iterative development process established a new desired session frequency of three to four months to enable full data analysis and algorithm iteration. The real-time availability of audio and video allowed the team to establish real-time reactionbased contingency planning. New standards were created to allow the crew to run tests sequentially, while concurrently enabling a success threshold. Feedback from the crew before, during, and after operations resulted in improvements to the procedures and information delivery methods. While work continues to improve the efficiency of the procedures, especially during the first operating session of a crew member, the SPHERES team has reached a stable operating environment which enables incremental technology maturation. This paper serves to document the lessons learned such that future space technology maturation projects may learn from the challenges faced, and possible solutions.

\section{Acknowledgements}

The authors wish to acknowledge the support from the DoD Space Technologies Program (STP) office and the NASA Johnson Space Center ISS Operations team, the NASA Marshall Space Flight Center astronaut office, and the JSC Reduced Gravity Office.

We thank the crews of ISS Expeditions 13, 14 and 15 for the many "Saturday Science" hours, as well as the multiple crews who attended SPHERES training.

We also thank the rest of the SPHERES team at the MIT SSL and PSI.

\footnotetext{
References

[1] Hilstad, Mark, "A Multi-Vehicle Testbed and Interface Framework for the Development and Verification of Separated Spacecraft Control Algorithms”. MS Thesis 2002 (SSL \#16-02)

[2] Saenz-Otero, A and D.W. Miller. "SPHERES: a platform for formation-flight research,” UV/Optical/IR Space Telescopes: Innovative Technologies and Concepts II conference, San Diego, CA, August 2005.

[3] Nolet, S., A. Saenz-Otero, D.W. Miller, and
} A. Fejzic, "SPHERES Operations Aboard 
the ISS: Maturation of GN\&C Algorithms in Microgravity," 30th Annual AAS Guidance and Control Conference, No. AAS 07-042, Breckenridge, Colorado, February 2007.

[4] Nolet, S. and D.W. Miller, "Autonomous docking experiments using the SPHERES testbed inside the ISS," Sensors and Systems for Space Applications, edited by R. T. Howard and R. D. Richards, Vol. 6555, SPIE, 2007.

[5] Nolet, S., "The SPHERES Navigation System: from Early Development to OnOrbit Testing," AIAA Guidance, Navigation and Control Conference and Exhibit, Hilton Head, South Carolina, 20-23 August 2007.

[6] Nolet, S. "Design of an Algorithm for Autonomous Docking With a Freely Tumbling Target.” Proc. of the SPIE Defense and Security Symposium 2005, Vol. 5799-16, Orlando, Florida, March 2005.

[7] Saenz-Otero, Alvar, "Design Principles for the Development of Space Technology Maturation Laboratories Aboard the International Space Station,” MIT Space Systems Laboratory No. 16-05, June, 2005

[8] Mandy C., A. Saenz-Otero, D. W. Miller, "Satellite Formation Flight and Realignment Maneuver Demonstration aboard the International Space Station", SPIE Optics + Photonics Conference 2007, San Diego, California, Aug. 26-30 2007.

[9] Mandy C., H. Sakamoto, A. Saenz-Otero and D. W. Miller, "Implementation of Satellite Formation Flight Algorithms Using SPHERES Aboard the International Space Station," 20th International Symposium on Space Flight Dynamics, Annapolis, Maryland, Sept. 24-28, 2007. 